\title{
Resultados Clínicos Iniciais do Primeiro Stent de Cromo-Cobalto Concebido no Brasil
}

\author{
Carlos Augusto Homem de Magalhães Campos ${ }^{1}$, Expedito E. Ribeiro' ${ }^{1}$, Pedro A. Lemos' ${ }^{1}$, Angel Obregon², \\ Henrique Ribeiro1, André G. Spadaro1, Eulógio E. Martinez', Luciano Almeida Fleury Curado', \\ Spero Penha Morato ${ }^{3}$, Cristina Nunes ${ }^{1}$, José Antônio Franchini Ramires ${ }^{1}$
}

\section{RESUMO}

Fundamentos: Os stents de cromo-cobalto são constituídos de hastes mais finas, apresentando maior flexibilidade e, possivelmente, menor proliferação neointimal que os stents de aço inoxidável. Método: O registro do stent Cronus (Scitech Produtos Médicos, Goiânia, GO) é um estudo prospectivo, não-randomizado, internacional, desenhado para avaliar a eficácia e a segurança do novo stent de cromo-cobalto de hastes finas em pacientes com insuficiência coronária. No total, 69 lesões foram tratadas em 53 pacientes envolvidos nessa análise. Resultados: Foi constatado sucesso do dispositivo em $98,5 \%$ dos casos. A perda luminal tardia foi de 0,7 $\pm 0,5 \mathrm{~mm}$, tendo ocorrido reestenose binária em $15 \%$ e revascularização do vasoalvo em $11,3 \%$ dos casos. A incidência de eventos cardíacos adversos maiores (morte, infarto do miocárdio ou revascularização do vaso-alvo) foi de $24,4 \%$ após $317 \pm 80$ dias de acompanhamento. Conclusão: Nossos resultados iniciais demonstram que o novo stent Cronus mostrou-se seguro e eficaz, com resultados clínicos semelhantes aos observados em outros stents de hastes finas.

DESCRITORES: Stents. Angioplastia transluminal percutânea coronária. Próteses e implantes.

\begin{abstract}
Initial Clinical Results of the First

Cobalt-Chromium Stent Designed in Brazil

Background: Thin-strut cobalt-chromium stents have greater flexibility and probably have less neointimal proliferation than stainless steel stents. Methods: The Cronus (Scitech Produtos Médicos, Goiânia, Brazil) registry is an international, non-randomized, prospective study designed to evaluate the efficacy and safety of a novel thin-strut cobalt-chromium stent in patients with coronary artery disease. A total of 69 lesions were treated in 53 patients included in this analysis. Results: The device success was $98.5 \%$. Mean late luminal loss was $0.7 \pm 0.5 \mathrm{~mm}$, binary restenosis occurred in $15 \%$ and target vessel revascularization in $11.3 \%$ of the cases. The incidence of major adverse cardiac events (death, myocardial infarction or target vessel revascularization) was $24.4 \%$ after $317 \pm 80$ days of follow-up. Conclusion: Our initial results demonstrate that the novel Cronus stent proved to be safe and effective, with clinical results similar to other available thin-strut stents.
\end{abstract}

DESCRIPTORS: Stents. Angioplasty, transluminal, percutaneous coronary. Prostheses and implants.
0 stents coronários de aço inoxidável 316L confeccionados por corte a laser predominaram na cardiologia intervencionista, sendo utilizados em mais de 90\% das intervenções coronárias percutâneas. No entanto, mais recentemente, stents confeccionados

Este estudo contou com o apoio institucional da Scitech Produtos Médicos (Brasil).

1 Instituto do Coração do Hospital das Clínicas da Faculdade de Medicina da USP - São Paulo, SP, Brasil.

${ }^{2}$ Centro de Investigaciones Médico-Quirúrgicas - CIMEQ - Havana, Cuba.

${ }^{3}$ Instituto de Pesquisas Energéticas e Nucleares da Universidade de São Paulo - São Paulo, SP, Brasil.

Correspondência: Carlos Augusto Campos. Hospital Ibiapaba - Serviço de Hemodinâmica e Cardiologia Intervencionista. Av. Dr. Jaime do Rego Macedo, 97 - Centro - Barbacena, MG, Brasil - CEP 36200-032 E-mail:cm-campos@uol.com.br

Recebido em: 24/6/2009 • Aceito em: 14/9/2009 a partir de ligas cromo-cobalto têm sido desenvolvidos com o intuito de melhorar algumas características dos stents tradicionais de aço inoxidável. Essas novas ligas apresentam maior resistência mecânica que o aço inoxidável e esse fato possibilita a confecção de stents com hastes mais finas, sem que haja, em tese, perda da força radial ou aumento do recoil do stent ${ }^{1-4}$. A redução da espessura das hastes possibilita a obtenção de stents mais flexíveis, com menor perfil de cruzamento e com evidências clínicas de menor proliferação neointimal em relação ao aço ${ }^{1,2}$.

O stent Cronus (Scitech Produtos Médicos, Goiânia, $\mathrm{GO})$ é um dispositivo constituído de liga de cromocobalto, de hastes finas, e que teve favorável avaliação pré-clínica previamente publicada ${ }^{5}$. Este registro procurou avaliar a segurança e a eficácia desse novo stent de concepção nacional. 


\section{MÉTODO}

Este é um estudo internacional, não-randomizado, prospectivo, para avaliação clínica do stent Cronus. Os pacientes foram incluídos no Instituto do Coração do Hospital das Clínicas da Faculdade de Medicina da Universidade de São Paulo (InCor-HCFMUSP) e no Centro de Investigaciones Médico-Quirúrgicas (CIMEQ - Havana, Cuba). O desfecho primário foi a ocorrência de eventos cardíacos adversos maiores (óbito cardíaco, infarto ou revascularização do vaso-alvo). Desfechos secundários incluíram sucesso imediato do dispositivo, perda luminal tardia, reestenose angiográfica e trombose.

\section{Stent em análise}

O stent Cronus foi construído a partir de tubo metálico em liga de cromo-cobalto L-605 cortado a laser, sendo, posteriormente, submetido a tratamento térmico e decapagem química e polido por eletroquímica seguida de passivação. O stent possui desenho em anéis corrugados, com espessura final das hastes de $75 \mu \mathrm{m}$. Em cada anel, o ângulo final máximo entre as hastes é de 45 graus para o stent expandido, formato especificamente concebido com o intuito de otimizar a força radial final. Os anéis foram construídos em células curtas $(1,1 \mathrm{~mm})$, o que permite a ampliação do número de células e aumenta a homogeneidade da cobertura metálica em segmentos tortuosos e angulados. A ligação interanel foi feita por meio de links curtos em formato sinusoide (espessura de $65 \mu \mathrm{m}$ ), que proporcionavam espaço circunferencial máximo de aproximadamente $6 \mathrm{~mm}$ de diâmetro com o intuito de permitir acesso a ramo lateral (Figura 1).

\section{Critérios de inclusão}

Pacientes com evidência clínica de angina do peito estável, instável, infarto agudo do miocárdio ou com prova funcional positiva, com tratamento percutâneo planejado, foram considerados para a inclusão. As lesões deveriam ser de novo, com obstrução luminal superior a 50\%. Foram permitidas abordagens em pacientes com acometimento coronário multiarterial e em enxertos de cirurgia de revascularização prévia.

\section{Procedimento}

A antiagregação plaquetária utilizada foi clopidogrel em dose de ataque de 300-600 mg (com recomendação para $600 \mathrm{mg}$ se o tempo para intervenção fosse inferior a seis horas), seguido de $75 \mathrm{mg} /$ dia por 30 dias. Além disso, o paciente recebeu prescrição para utilizar aspirina (100-300 mg/dia) indefinidamente. Após obtenção de acesso vascular (maior ou igual a 6 F) e introdução do cateter, foi administrada heparina nas doses preconizadas para obter tempo de coagulação ativada > 250 segundos (ou > 200 segundos se houvesse utilização de inibidores da glicoproteína IIb/IIla). Foi selecionado um stent de tamanho adequado para tratar a lesão. Stents de 2,5 mm, 3,0 mm e 3,5 mm de diâmetro e de $9 \mathrm{~mm}, 13 \mathrm{~mm}, 16 \mathrm{~mm}, 19 \mathrm{~mm}, 23 \mathrm{~mm}$ e $28 \mathrm{~mm}$ de comprimento estavam disponíveis para uso. A utilização de pré-dilatação com balão ou de stent direto ficou a critério do operador.

\section{Coleta e análise de dados}

Os dados da evolução intra-hospitalar foram coletados por médicos treinados, durante a internação índice, seguindo o preenchimento de formulários previamente padronizados. A coleta incluiu características clínicas, resultados de exames laboratoriais, dados do procedimento invasivo (desde tempo até características angiográficas e da intervenção) e evolução clínica até a alta hospitalar. A coleta de informações sobre a evolução tardia foi realizada por seguimento ambulatorial realizado nos centros envolvidos, revisão dos registros hospitalares ou contato telefônico.

Todas as angiografias foram analisadas pelo Serviço de Hemodinâmica e Cardiologia Intervencionista do InCor, avaliando as angiografias inicial e de seguimento para determinar o sucesso do procedimento, medidas e características do vaso, e eventos adversos angiográficos. A aquisição de imagens foi realizada usando duas ou mais projeções angiográficas da estenose após a administração de nitrato. Essas projeções foram repetidas no momento da angiografia de seguimento. Os cineangiogramas foram analisados por um observador cego quanto ao stent empregado. As características morfológicas qualitativas foram avaliadas utilizando critérios padronizados. O diâmetro luminal mínimo da lesão, sua extensão e os diâmetros dos segmentos de referência foram medidos antes e após o implante do stent e ao reestudo angiográfico. O reestudo angiográfico foi realizado entre seis e nove meses após o procedimento índice. A análise quantitativa das artérias coronárias foi realizada com o emprego do sistema CAAS-II (Pie Medical Imaging, Maastricht, Holanda).

As variáveis contínuas foram descritas como média \pm desvio padrão ou mediana e intervalo interquartil, e variáveis categóricas foram representadas como sua porcentagem.

\section{Definições}

Foi considerado óbito cardíaco qualquer mortalidade decorrente de infarto do miocárdio, perfuração cardíaca ou tamponamento pericárdico, arritmia ou distúrbio de condução, acidente vascular cerebral, complicações do procedimento ou qualquer óbito em que uma causa cardíaca não pôde ser afastada. Óbito nãocardíaco foi classificado como morte por qualquer outra causa que não as cardíacas. Infarto agudo do miocárdio após procedimento foi definido como elevação de creatina quinase fração $M B(C K-M B)$ maior ou igual a três vezes o valor de referência. Revascularização do vaso-alvo foi caracterizada por nova intervenção, percutânea ou cirúrgica, no vaso abordado no procedimento índice. Reestenose angiográfica bi- 

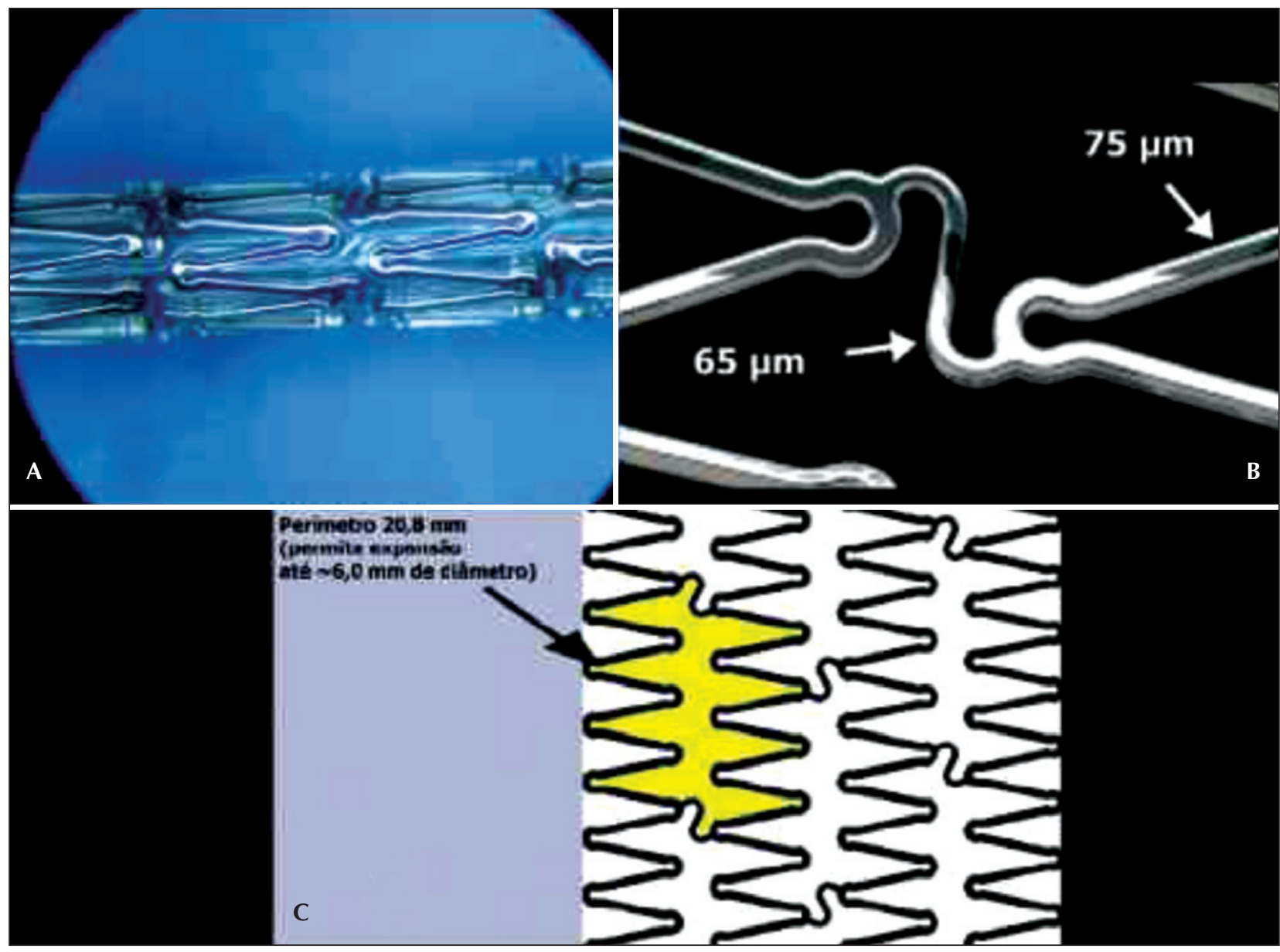

Figura 1 - Em A, fotografia em grande aumento do stent metálico de liga de cromo-cobalto L-605, após os polimentos elétrico e químico. Observa-se a angulação inter-hastes inferior a 45 graus (45 graus somente atingido como ângulo final máximo do stent expandido a 4,5 mm de diâmetro). Em B, detalhe das hastes do stent (espessura de $75 \mu \mathrm{m}$ ) e do link interanel em formato sinusoide (espessura de 65 m). Em C, esquema ilustrativo do espaço circunferencial lateral do stent (acesso a ramo lateral).

nária foi definida pela presença de estenose em diâmetro maior que $50 \%$ ao reestudo angiográfico. Sucesso do dispositivo foi considerado quando se obteve estenose residual $<30 \%$ na lesão-alvo, com fluxo final TIMI III (Trombolysis In Myocardial Infarction), com posicionamento adequado do stent na lesão e sua apropriada dilatação.

A perda luminal tardia foi definida como sendo diâmetro luminal mínimo imediatamente após o implante do stent menos o diâmetro luminal mínimo observado no reestudo angiográfico.

A presença de trombose do stent foi determinada de acordo com os critérios do Academic Research Consortium $^{4}$ quanto a:

- Tempo de ocorrência:

a) aguda: ocorrida dentro das primeiras 24 horas;

b) subaguda: entre o primeiro e o trigésimo dias após implante; c) tardia: do trigésimo dia até o final do primeiro ano após implante;

d) muito tardia: após o primeiro ano do implante.

- Grau de certeza:

a) definitiva: confirmação angiográfica ou por meio de necropsia de trombose no vaso-alvo;

b) provável: qualquer óbito não explicado nos primeiros 30 dias após o procedimento ou qualquer infarto do miocárdio no território relacionado ao vaso tratado, sem confirmação angiográfica;

c) possível: qualquer óbito não explicado após 30 dias do implante.

\section{RESULTADOS}

No período de fevereiro de 2007 a dezembro de 2008, 53 pacientes foram tratados com 69 stents de 
liga de cromo-cobalto (média de 1,3 $\pm 0,49$ stent/ paciente). O quadro clínico de admissão foi de angina estável em 75,5\%, com média de idade de 62 anos, sendo $66,1 \%$ dos pacientes do sexo masculino, $26,1 \%$ de diabéticos, e a maioria $(64,1 \%)$ apresentando acometimento coronário uniarterial (Tabela 1). As lesões eram, em grande parte, do tipo B2/C (53,7\%), com diâmetro de referência de $2,7 \pm 0,7 \mathrm{~mm}$ e comprimento médio de 16,7 $\pm 3,5 \mathrm{~mm}$, sendo abordadas, em sua maioria, coronárias nativas $(98 \%)$, com $2,8 \%$ de utilização em enxertos venosos (Tabela 2).

\section{Desfechos hospitalares}

Foi constatado sucesso do dispositivo em 98,5\% dos casos $(n=68)$. Em um caso o stent não conseguiu ultrapassar a lesão, sendo tentados outros dois tipos de stent disponíveis no mercado, também sem sucesso. A técnica de implante utilizada foi de stent direto em $73 \%$ das abordagens. No período hospitalar foram constatados três $(5,6 \%)$ casos de infarto após procedimento, sem surgimento de onda $\mathrm{Q}$ ao eletrocardiograma ou repercussões clínicas. Não houve, durante internação, ocorrência de óbito ou revascularização do vaso-alvo de urgência.

\section{Desfechos tardios}

Foi obtido seguimento clínico de 98,1\% dos pacientes $(n=52)$ por um período médio de $317 \pm 80$ dias.

\section{TABELA 1}

Características clínicas dos pacientes $(n=53)$

\begin{tabular}{lc} 
Média de idade, anos & $62 \pm 13$ \\
Sexo masculino, \% & 66,0 \\
Diabetes melito, \% & 26,1 \\
Tabagismo atual, \% & 16,9 \\
Dislipidemia, \% & 90,5 \\
Hipertensão arterial, \% & 88,6 \\
IAM prévio, \% & 30,1 \\
ICP prévia,\% & 13,2 \\
CRVM prévia, \% & 9,4 \\
ICC, \% & 13,2 \\
Padrão de acometimento coronário, \% & \\
$\quad$ Uniarterial & 64,1 \\
Biarterial & 32,2 \\
Triarterial & 3,7 \\
Quadro clínico de admissão, \% & \\
$\quad$ Angina estável & 75,5 \\
SCA sem supradesnível de ST & 17 \\
IAM com supradesnível do segmento ST & 7,5 \\
\hline
\end{tabular}

IAM = infarto agudo do miocárdio; ICC = insuficiência cardíaca congestiva; ICP = intervenção coronária percutânea; CRVM = cirurgia de revascularização miocárdica; $\mathrm{SCA}=$ síndrome coronária aguda.
Nesse período, a ocorrência de eventos cardíacos adversos maiores foi de $24,4 \%$, sendo $11,3 \%$ de revascularização do vaso-alvo, 5,6\% de infarto não-fatal, e $7,5 \%$ de óbitos $(n=4)$. Vale ressaltar que todos os infartos não-fatais ocorreram no período periprocedimento e foram constituídos por infartos sem onda $Q$ e sem repercussões clínicas, como citado anteriormente. Com relação aos óbitos, houve dois casos de morte de origem cardíaca, ocasionada por possível trombose (morte súbita após 30 dias de implante) e trombose provável (morte súbita antes dos 30 dias de implante). Dois pacientes faleceram por complicações secundárias a quadros de broncopneumonia (Tabela 3), sem

TABELA 2

Características angiográficas

$\begin{array}{lc}\text { Complexidade ACC/AHA, \% } & \\ \text { A } & 13 \\ \text { B1 } & 33,3 \\ \text { B2/C } & 53,7 \\ \text { Vaso tratado, \% } & \\ \text { DA } & 34,7 \\ \text { CX } & 34,7 \\ \text { CD } & 27,8 \\ \text { Enxerto venoso } & 2,8 \\ \text { Diâmetro de referência, mm } & 2,7 \pm 0,7 \\ \text { Diâmetro luminal mínimo, mm } & 1,1 \pm 0,4 \\ \text { Diâmetro de estenose (pré), \% } & 61 \pm 11 \\ \text { Comprimento, mm } & 16,7 \pm 3,5\end{array}$

$\mathrm{ACC} / \mathrm{AHA}=$ American College of Cardiology/American Heart Association; $\mathrm{CD}=$ artéria coronária direita; $\mathrm{CX}=$ artéria circunflexa; DA = descendente anterior.

TABELA 3

Resultados clínicos e angiográficos tardios

$\begin{array}{lc}\text { Eventos clínicos, \% } & \\ \text { ECAM } & 24,4 \\ \text { RVA } & 11,3 \\ \text { IAM não-fatal } & 5,6 \\ \text { Óbito } & 7,5 \\ \text { Óbito cardíaco } & 3,75 \\ \text { Óbito não-cardíaco } & 3,75 \\ \text { Resultados angiográficos tardios } & \\ \text { DLM, mm } & 1,9 \pm 0,6 \\ \text { Diâmetro de estenose, \% } & 29 \pm 18,6 \\ \text { Perda luminal tardia, mm } & 0,7 \pm 0,5 \\ \text { Estenose angiográfica, \% } & 15\end{array}$

DLM = diâmetro luminal mínimo; ECAM = eventos cardíacos adversos maiores; IAM = infarto agudo do miocárdio; $\mathrm{RVA}=$ revascularização do vaso-alvo. 
TABELA 4

Comparação de desfechos angiográficos entre diferentes stents de cromo-cobalto

\begin{tabular}{lccc}
\hline & Driver $^{\mathrm{TM}}$ (Medtronic) & Multi-Link Vision $^{\mathrm{TM}}$ (Guidant) & Cronus (Scitech) \\
\hline Perda luminal tardia, $\mathrm{mm}$ & $0,94 \pm 0,54$ & $0,83 \pm 0,56$ & $0,7 \pm 0,5$ \\
Reestenose angiográfica, \% & 15,7 & 15,7 & 15 \\
\hline
\end{tabular}

correlação com o procedimento índice. Do total, 31 $(58,5 \%)$ pacientes concordaram em participar do reestudo angiográfico. Nessa população, a reestenose angiográfica ocorreu em $15 \%$, com $11,3 \%$ de novas revascularizações do vaso-alvo. O diâmetro luminal mínimo foi de 1,9 $\pm 0,6 \mathrm{~mm}$, com diâmetro de estenose médio de $29 \pm 18,6 \%$ e perda luminal tardia de $0,7 \pm$ $0,5 \mathrm{~mm}$ (Tabela 3).

\section{DISCUSSÃO}

Este registro foi realizado para determinar o comportamento clínico do primeiro stent metálico de cromo-cobalto desenvolvido no Brasil. Nesta amostra inicial, o stent Cronus mostrou-se seguro e eficaz para o tratamento de lesões arteriais coronárias.

O dispositivo foi implantado com alto índice de sucesso $(98,5 \%)$ em uma população de mundo real, com grande proporção de lesões complexas (tipo B2/C). Esses resultados são similares aos de outros dispositivos de aço ou de cromo-cobalto avaliados em estudos prévios $(87-100 \%)^{3,4,6-9}$.

Com relação à segurança, o stent Cronus apresentou bom perfil de segurança, demonstrando taxa satisfatória de ocorrência de eventos cardíacos adversos maiores. Os índices de infarto não-fatais ficaram restritos à elevação de marcadores de necrose miocárdica periprocedimento. A ocorrência de dois $(3,75 \%)$ possíveis óbitos cardíacos ao final de mais de 300 dias de seguimento clínico é compatível com a população em estudo e demonstra a segurança do dispositivo. Em uma série do registro InCor-HCFMUSP, incluindo 2.290 pacientes tratados com stent convencional, a mortalidade tardia documentada foi de $6,8 \%{ }^{10}$. Estabelecendo-se um paralelo com estudo semelhante com stent convencional de cromo-cobalto, em pacientes com diâmetro do vaso médio inferior a $3 \mathrm{~mm}$, a mortalidade ao final de um ano foi de $5 \%{ }^{11}$.

Como limitação deste estudo, podemos citar o baixo índice de reestudo angiográfico (58,5\%). Esse fato decorreu da baixa aceitação por parte dos pacientes, quando assintomáticos, em realizar novo procedimento invasivo. Nesse grupo, encontramos perda luminal tardia muito satisfatória para um stent metálico convencional $(0,7 \mathrm{~mm})$. No entanto, como obtivemos seguimento clínico tardio médio superior a 300 dias na quase totalidade dos pacientes $(98,1 \%)$ e baixa incidência de revascularização do vaso-alvo guiada por isquemia $(11,3 \%)$, podemos inferir que a perda luminal tardia encontrada tem boa correlação com a população global em estudo. Por tratar-se de uma população com $26,1 \%$ de diabéticos, comprimento médio das lesões de $16,7 \pm 3,5 \mathrm{~mm}$ e diâmetro de referência médio de $2,7 \pm 0,7 \mathrm{~mm}$, pouco mais de $10 \%$ de novas reintervenções correspondem a resultados favoráveis em se tratando de um stent metálico convencional. Como pode ser observado na Tabela 4, os desfechos de eficácia são semelhantes aos de outros stents de cromo-cobalto, como os stents Dri$\operatorname{ver}^{\mathrm{TM}}$ (Medtronic, Minneapolis, Estados Unidos) ou Multi-Link Vision ${ }^{\mathrm{TM}}$ (Guidant, Santa Clara, Estados Unidos) $)^{3,4,9,12}$.

Finalmente, a importância desse dispositivo reside no fato de sua engenharia, seu desenvolvimento préclínico e sua avaliação clínica terem sido realizados no Brasil, com achados semelhantes aos de estudos internacionais, sendo esta uma análise pioneira nesse sentido.

\section{CONCLUSÃO}

Nossos resultados iniciais demonstram que o stent Cronus mostrou-se seguro, com bons resultados angiográficos e alta porcentagem de sucesso do dispositivo.

\section{CONFLITO DE INTERESSES}

Os autores declararam inexistência de conflito de interesses.

\section{REFERÊNCIAS BIBLIOGRÁFICAS}

1. Kastrati A, Mehilli J, Dirschinger J, Dotzer F, Schühlen H, Neumann FJ, et al. Intracoronary stenting and angiographic results: strut thickness effect on restenosis outcome (ISARSTEREO) trial. Circulation. 2001;103(23):2816-21.

2. Pache J, Kastrati A, Mehilli J, Schühlen H, Dotzer F, Hausleiter J, et al. Intracoronary stenting and angiographic results: strut thickness effect on restenosis outcome (ISAR-STEREO-2) trial. J Am Coll Cardiol. 2003;41(8):1283-8.

3. Legrand V, Kelbaek H, Hauptmann KE, Glogar D, Rutsch W, Grollier G, et al. Clinical and angiographic analisys with a cobalt alloy coronary stent (driver) in stable and unstable angina pectoris. Am J Cardiol. 2006;97(3):349-52.

4. Sketch MH Jr, Ball M, Rutherford B, Popma JJ, Russell C, Kereiakes DJ; Driver investigators. Evaluation of the Medtronic (Driver) cobalt-chromium alloy coronary stent system. Am J Cardiol. 2005;95(1):8-12. 
5. Lemos PA, Laurindo FRM, Morato SP, Takimura C, Campos CA, Gutierrez PS, et al. Stent coronário de liga cromocobalto concebido no Brasil: achados histológicos preliminares em modelo experimental porcino. Rev Bras Cardiol Invas. 2007;15(4):378-85.

6. Martínez-Elbal L, Ruiz-Nodar JM, Zueco J, López-Minguez JR, Moreu J, Calvo I, et al. Direct coronary stenting versus stenting with balloon pre-dilation: immediate and follow-up results of a multicentre, prospective, randomized study. The DISCO trial. DIrect Stenting of COronary Arteries. Eur Heart J. 2002;23(8):633-40.

7. Baim DS, Flatey M, Caputo R, O'Shaughnessy C, Low R, Fanelli C, et al.; PRE-Dilatation vs Direct Stenting In Coronary Treatment (PREDICT) Trial. Comparison of PRE-dilatation vs direct stenting in coronary treatment using the Medtronic AVE S670 Coronary Stent System (the PREDICT trial). Am J Cardiol. 2001;88(12):1364-9.

8. Ijsselmuiden AJ, Serruys PW, Scholte A, Kiemeneij F, Slagboom $\mathrm{T}$, vd Wieken LR, et al. Direct coronary stent implantation does not reduce the incidence of in-stent restenosis or major adverse cardiac events: six month results of a randomized trial. Eur Heart J. 2003;24(5):421-9.

9. Brito FS Jr, Caixeta AM, Perin MA, Rati M, Arruda JA, Cantarelli $M$, et al.; DIRECT Study Investigators. Comparison of direct stenting versus stenting with predilation for the treatment of selected coronary narrowings. Am J Cardiol. 2002;89(2):115-20.

10. Campos CA, Ribeiro EE, Lemos PA, Kajita LJ, Esteves A, Gama $M N$, et al. Impacto do uso de stents farmacológicos sobre a mortalidade tardia. Apresentação pessoal: XXIX Congresso da SBHCl; junho de 2007.

11. Strehblow C, Gyöngyösi M, Zenker G, Wallner H, Heigert $M$, Siostrzonek $P$, et al. Small vessel stenting with cobaltchromium stents (Arthos Pico) in a real world setting. Coron Artery Dis. 2007;18(4):305-11.

12. Kereiakes DJ, Cox DA, Hermiller JB, Midei MG, Bachinsky WB, Nukta ED, et al.; Guidant Multi-Link Vision Stent Registry Investigators. Usefulness of a cobalt chromium coronary stent alloy. Am J Cardiol. 2003;92(4):463-6. 